\title{
Creation of a chemical-technological system digital twin using the Python language
}

\author{
M. Dli ${ }^{1,2}$, E. Vlasova ${ }^{2}$, A. Sokolov ${ }^{3}$, E. Morgunova ${ }^{3}$ \\ ${ }^{1}$ Branch of the National Research University "MPEl" in Smolensk, Smolensk, Russia \\ 2 Synergy University, Moscow, Russia \\ ${ }^{3}$ National Research University "MPEI", Moscow, Russia \\ *ansokol98@mail.ru
}

\begin{abstract}
Currently, when modeling complex technological processes in cyber-physical systems, procedures for creating so-called "digital twins" (DT) have become widespread. DT are virtual copies of real objects which reflect their main properties at various stages of the life cycle. The use of digital twins allows real-time monitoring of the current state of the simulated system, and also provides additional opportunities for engineering and deeper customization of its components to improve the quality of products. The development of the "digital twin" technology is facilitated by the ongoing Fourth Industrial Revolution, which is characterized by the massive introduction of cyber-physical systems into production process. These systems are based on the use of the latest technologies for data processing and presentation and have a complex structure of information chain between its components. When creating digital twins of such systems elements, it is advisable to use programming languages, that allow visualization of simulated processes and provide a convenient and developed apparatus for working with complex mathematical dependencies. The Python programming language has similar characteristics. In the article, as an example of a cyberphysical system, a chemical-technological system based on a horizontal-grate machine is considered. This system is designed to implement the process of producing pellets from the apatite-nepheline ore mining wastes. The article describes various aspects of creating a digital twin of its elements that carry out the chemical-technological drying process in relation to a single pellet. The digital twin is implemented using the Python 3.7.5 programming language and provides the visualization of the process in the form of a three-dimensional interactive model. Visualization is done using the VPython library. The description of the digital twin software operation algorithm is given, as well as the type of the information system interface, the input and output information type, the results of modeling the investigated chemical-technological process. It is shown that the developed digital twin can be used in three versions: independently (Digital Twin Prototype), as an instance of a digital twin (Digital Twin Instance), and also as part of a digital twins set (Digital Twin Aggregate).
\end{abstract}

Keywords: digital twin, Python, cyber-physical systems, man-made waste processing, modeling of chemical technological processes

For citation: Dli M., Vlasova E., Sokolov A., Morgunova E. Creation of a chemical-technological system digital twin using the Python language. Prikladnaya informatika=Journal of Applied Informatics, 2021, vol.16, no.1, pp.22-31. DOI: 10.37791/2687-0649-2021-16-1-22-31 


\title{
Создание цифрового двойника химико-технологической системы с использованием языка Python
}

\author{
М. И. Дли ${ }^{1,2}$, Е. А. Власова ${ }^{2}$, А. М. Соколов ${ }^{3}$, Э. В. Моргунова ${ }^{3}$ \\ 1 Филиал ФГБОУ ВО «Национальный исследовательский университет "МЭИ"» в г. Смоленске, \\ Смоленск, Россия \\ 2 Университет «Синергия», Москва, Россия \\ 3 ФГБОУ ВО «Национальный исследовательский университет "МЭИ"», Москва, Россия \\ *ansokol98@mail.ru
}

\begin{abstract}
Аннотация. В настоящее время при моделировании сложных технологических процессов в киберфизических системах все более широкое распространение получают процедуры создания так называемых цифровых двойников (ЦД), которые являются виртуальными копиями реальных объектов и отражают их основные свойства и характеристики на различных этапах жизненного цикла. Применение цифровых двойников позволяет в реальном времени отслеживать текущее состояние моделируемой системы, а также предоставляет дополнительные возможности для инжиниринга и более глубокой настройки входящих в нее компонентов для повышения уровня качества выпускаемой продукции. Развитию технологии «цифровой двойник» способствует происходящая в настоящее время Четвертая промышленная революция, характеризующаяся массовым внедрением в производство киберфизических систем. Указанные системы основаны на использовании новейших технологий обработки и представления данных и обладают сложной структурой информационных связей между ее компонентами. При создании цифровых двойников элементов подобных систем целесообразно использовать языки программирования, которые позволяют реализовывать визуализацию моделируемых процессов, а также предоставляют удобный и развитый аппарат для работы со сложными математическими зависимостями. Подобными характеристиками обладает язык программирования Python.

В статье в качестве примера киберфизической системы рассматривается химикотехнологическая система на основе обжиговой машины конвейерного типа. Данная система предназначена для реализации процесса производства окатышей из отходов добычи апатитнефелиновых руд. В статье описаны различные аспекты создания цифрового двойника ее элементов, осуществляющих химико-технологический процесс сушки применительно к единичному окатышу. Цифровой двойник реализован с использованием языка программирования Python 3.7.5, и предусматривает визуализацию протекания процесса в виде трехмерной интерактивной модели. Визуализация выполнена с применением библиотеки VPython. Приводится описание алгоритма работы программного обеспечения цифрового двойника, вид интерфейса информационной системы, формат входной и выходной информации, а также результаты моделирования исследуемого химико-технологического процесса. Показано, что разработанный цифровой двойник может применяться в трех вариантах: самостоятельно (Digital Twin Prototype), в качестве экземпляра цифрового двойника (Digital Twin Instance), а также в составе совокупности цифровых двойников (Digital Twin Aggregate).
\end{abstract}

Ключевые слова: цифровой двойник, Руthon, киберфизические системы, переработка техногенных отходов, моделирование химико-технологических процессов

Для цитирования: Дли М. И., Власова Е. А., Соколов А. М., Моргунова Э. В. Creation of a chemicaltechnological system digital twin using the Python language // Прикладная информатика. 2021. Т. 16. № 1. C. 22-31. DOI: 10.37791/2687-0649-2021-16-1-22-31 